\title{
Weaknesses of a Dynamic ID Based Remote User Authentication Protocol for Multi-Server Environment
}

\author{
R. Madhusudhan, Adireddi Praveen \\ Department of Mathematical \& Computational Sciences, National Institute of Technology Karnataka, Surathkal, \\ India \\ Email: madhu_nitks@yahoo.com
}

Received December 2013

\begin{abstract}
Currently, smart card based remote user authentication schemes have been widely adopted due to their low cost and convenient portability. With the purpose of using various different internet services with single registration and to protect the users from being tracked, various dynamic ID based multi-server authentication protocols have been proposed. Recently, Li et al. proposed an efficient and secure dynamic ID based authentication protocol using smart cards. They claimed that their protocol provides strong security. In this paper, we have demonstrated that Li et al.'s protocol is vulnerable to replay attack, denial of service attack, smart card lost attack, eavesdropping attack and server spoofing attacks.
\end{abstract}

\section{Keywords}

\section{Authentication; Smart Card; Dynamic ID; Multi-Server Environments; Password}

\section{Introduction}

With the rapid growth of internet technologies and mobile communication services, remote user authentication is being more and more critical in order to prevent access to illegal users. Password based authentication is one of the simplest and the most convenient authentication mechanisms over remote access networks but it is not secure over insecure communication channels. Hence a large number of smart card based authentication protocols have been proposed to overcome the drawbacks of traditional password based protocols. These can be categorized as static ID [1-3] and dynamic ID based protocols. To achieve user's anonymity, dynamic ID based authentication techniques [4-6] have been developed by many researchers.

In general, an efficient remote user authentication protocol should satisfy some functional and security requirements [7-11]. Based on the use of environment, authentications protocols can be divided into two categories: single-server and multi-server environments. Multi server architecture [12-15] provides the flexibility of using single registration across various different networks.

In 2009, Liao and Wang [16] proposed a dynamic ID based authentication protocol for multi-server environ- 
ments. Hsiang and Shih [17] found that Liao-Wang's protocol is vulnerable to insider's attack, masquerade attack, server spoofing attack, registration center spoofing attack. To overcome these weaknesses Hsiang and Shih proposed an improved protocol. But Lee et al. [18] found that this protocol is also not secure and susceptible to masquerade attack and server spoofing attack. To overcome the weaknesses of Hsiang and Shih's protocol, Lee et al. proposed an improved protocol and claimed that their protocol can resist all kinds of attacks.

In 2013, Li et al. [19] found that Lee et al.'s protocol is vulnerable to forgery attack, server spoofing attack and proposed a dynamic ID based authentication protocol for multi-server environments. They claimed that it is secure and can resist various attacks. However, in this paper we have demonstrated that $\mathrm{Li}$ et al.'s protocol is vulnerable to replay attack, denial of service attack, smart card lost attack, eavesdropping attack and server spoofing attacks.

The rest of this paper is organized as follows. In Section 2, we have given a brief review on Li et al.'s protocol. Section 3 provides the cryptanalysis of Li et al.'s protocol. Finally we conclude this paper in Section 4.

\section{Review of Li et al.'s Protocol}

The notations used in this paper are described in Table $\mathbf{1}$.

$\mathrm{Li}$ et al.'s protocol contains three participants, the user $\mathrm{U}_{\mathrm{i}}$, the server $\mathrm{S}_{\mathrm{j}}$, and the registration center RC. RC chooses the master secret key $\mathrm{x}$ and a secret number $\mathrm{y}$ to compute $\mathrm{h}(\mathrm{x} \| \mathrm{y})$ and $\mathrm{h}\left(\mathrm{SID}_{\mathrm{j}} \| \mathrm{h}(\mathrm{y})\right)$, and then shares them with $S_{j}$ via a secure channel. There are four phases in the protocol: registration phase, login phase, verification phase, and password change phase.

\subsection{Registration Phase}

When the user $U_{i}$ wants to access the services, the user $U_{i}$ and the registration center RC need to perform the following steps to finish the registration phase:

1) $U_{i}$ freely chooses his identity $I D_{i}$, the password $P W_{i}$, and computes $A_{i}=h\left(b \oplus P W_{i}\right)$, where b is a random number generated by $U_{i}$. Then $U_{i}$ sends ID and $A_{i}$ to the registration center RC for registration through a secure channel.

2) Now, Registration center, RC computes $B_{i}=h\left(I_{i} \| x\right), C_{i}=h\left(I D_{i}\|h(y)\| A_{i}\right), D_{i}=h\left(B_{i} \| h(x \| y)\right), E_{i}=B_{i} \oplus$ $\mathrm{h}(\mathrm{x} \| \mathrm{y})$. RC stores $\left\{\mathrm{C}_{\mathrm{i}}, \mathrm{D}_{\mathrm{i}}, \mathrm{E}_{\mathrm{i}}, \mathrm{h}(),. \mathrm{h}(\mathrm{y})\right\}$ on the user's smart card and sends it to user $\mathrm{U}_{\mathrm{i}}$ via a secure channel.

3) User keys b into smart card and finally it contains $\left\{\mathrm{C}_{\mathrm{i}}, \mathrm{D}_{\mathrm{i}}, \mathrm{E}_{\mathrm{i}}, \mathrm{b}, \mathrm{h}(),. \mathrm{h}(\mathrm{y})\right\}$.

\subsection{Login Phase}

Whenever $U_{i}$ wants to login $S_{j}$, he must perform the following steps to generate a login request message:

Table 1. Notations used.

\begin{tabular}{cc}
\hline $\mathrm{U}_{\mathrm{i}}$ & The ith user \\
\hline $\mathrm{ID}_{\mathrm{i}}$ & The identity of $\mathrm{U}_{\mathrm{i}}$ \\
$\mathrm{PW}_{\mathrm{i}}$ & The password of $\mathrm{U}_{\mathrm{i}}$ \\
$\mathrm{S}_{\mathrm{j}}$ & The jth server \\
$\mathrm{RC}$ & Registration center \\
$\mathrm{SID}_{\mathrm{j}}$ & Identity of $\mathrm{S}_{\mathrm{j}}$ \\
$\mathrm{CID}_{\mathrm{i}}$ & dynamic ID of $\mathrm{U}_{\mathrm{i}}$ \\
$\mathrm{x}$ & master secret key maintained by registration center \\
$\mathrm{y}$ & secret number generated by RC \\
$\mathrm{h}()$. & one-way hash function \\
$\oplus$ & Exclusive-or operation \\
$\|$ & Message concatenation operation \\
$\rightarrow$ & A common channel \\
$\Rightarrow$ & A secure channel \\
\hline
\end{tabular}


1) $U_{i}$ inserts his smart card into the card reader and inputs $I_{i}$ and $P W_{i}$. Then the smart card computes $A_{i}=$ $\mathrm{h}\left(\mathrm{b} \oplus \mathrm{PW}_{\mathrm{i}}\right), \mathrm{C}_{\mathrm{i}}^{*}=\mathrm{h}\left(\mathrm{ID}_{\mathrm{i}}\|\mathrm{h}(\mathrm{y})\| \mathrm{A}_{\mathrm{i}}\right)$, and checks whether the computed $\mathrm{C}_{\mathrm{i}}^{*}$ is equal to $\mathrm{C}_{\mathrm{i}}$. If they are equal, $\mathrm{U}_{\mathrm{i}}$ proceed the following steps. Otherwise the smart card aborts the session.

2) The smart card generates a random number $N_{i}$ and computes $P_{i j}=E_{i} \oplus h\left(h\left(\operatorname{SID}_{j} \| h(y)\right) \| N_{i}\right), C_{1} D_{i}=A_{i} \oplus$ $\mathrm{h}\left(\mathrm{D}_{\mathrm{i}}|| \mathrm{SID}_{\mathrm{j}} \| \mathrm{N}_{\mathrm{i}}\right), \mathrm{M}_{1}=\mathrm{h}\left(\mathrm{P}_{\mathrm{ij}}|| C \mathrm{CI}_{\mathrm{i}}|| \mathrm{D}_{\mathrm{i}}|| \mathrm{Ni}\right)$ and $\mathrm{M}_{2}=\mathrm{h}\left(\mathrm{SID}_{\mathrm{j}} \| \mathrm{h}(\mathrm{y})\right) \oplus \mathrm{N}_{\mathrm{i}}$.

3) $U_{i}$ submits $\left\{P_{i j}, C I D_{i}, M_{1}, M_{2}\right\}$ to $S_{j}$ as a login request message.

\subsection{Verification Phase}

After $S_{j}$ receiving the login message $\left\{P_{i j}, C_{D} D_{i}, M_{1}, M_{2}\right\}, S_{j}$ and $U_{i}$ perform the following steps for mutual authentication and session key agreement.

1) $S_{j}$ computes $N_{i}=M_{2} \oplus h\left(\operatorname{SID}_{j} \| h(y)\right), E_{i}=P_{i j} \oplus h\left(h\left(\operatorname{SID}_{j} \| h(y)\right) \| N_{i j}\right), B_{i}=E_{i} \oplus h(x \| y), D_{i}=h\left(B_{i} \| h(x \| y)\right)$ and $\mathrm{A}_{\mathrm{i}}=\mathrm{CID}_{\mathrm{i}} \oplus \mathrm{h}\left(\mathrm{D}_{\mathrm{i}}\left\|\operatorname{SID}_{\mathrm{j}}\right\| \mathrm{N}_{\mathrm{i}}\right)$ by using $\left\{\mathrm{P}_{\mathrm{ij}}, \mathrm{CID}_{\mathrm{i}}, \mathrm{M}_{1}, \mathrm{M}_{2}\right\}$, h(SID $\left.\left.\operatorname{SI}_{\mathrm{j}} \| \mathrm{y}\right)\right)$ and $\mathrm{h}(\mathrm{x} \| \mathrm{y})$.

2) $S_{j}$ computes $h\left(P_{i j} / / C I D_{i} / / D_{i} / / N_{i}\right)$ and checks whether it is equal to $M_{1}$. If they are not equal, $S_{j}$ rejects the login request and terminates this session. Otherwise, $S_{j}$ accepts the login request message. Then $S_{j}$ generates a nonce $N_{j}$ and computes $M_{3}=h\left(D_{i}\left\|A_{i}\right\| N_{j} \| S I D_{j}\right), M_{4}=A_{i} \oplus N_{i} \oplus N_{j}$. Finally, $S_{j}$ sends the message $\left\{M_{3}, M_{4}\right\}$ to $\mathrm{U}_{\mathrm{i}}$.

3) After receiving the response message $\left\{M_{3}, M_{4}\right\}$ sent from $S_{j}$, $U_{i}$ computes $N_{j}=A_{i} \oplus N_{i} \oplus M_{4}, h\left(D_{i}\left\|A_{i}\right\|\right.$ $\mathrm{N}_{\mathrm{j}} \| \mathrm{SID}_{\mathrm{j}}$ ) and checks this with the received message $\mathrm{M}_{3}$. If they are equal, $\mathrm{U}_{\mathrm{i}}$ successfully authenticates $\mathrm{S}_{\mathrm{j}}$. Then, the user $U_{i}$ computes the mutual authentication message $M_{5}=h\left(D_{i}|| A_{i}|| N_{i}|| S I D_{j}\right)$ and sends $\left\{M_{5}\right\}$ to the server $S_{j}$.

4) Upon receiving the message $\left\{M_{5}\right\}, S_{j}$ computes $h\left(D_{i}|| A_{i}|| N_{i}|| S_{I} D_{j}\right)$ and checks it with the received message $\left\{M_{5}\right\}$. If they are equal, $S_{j}$ authenticates $U_{i}$. User $U_{i}$ and the server $S_{j}$ compute $S K=h\left(D_{i}|| A_{i}|| N_{i}|| N_{j}|| S I D_{j}\right)$, which is taken as their session key for further communication.

\subsection{Password Change Phase}

This phase is invoked whenever $\mathrm{U}_{\mathrm{i}}$ wants to change his password $\mathrm{PW}_{\mathrm{i}}$ to a new password $\mathrm{PW}_{\text {new }}$. There is no need for a secure channel for password change and it can be finished without communicating with the registration center RC.

1) $U_{i}$ inserts smart card into the card reader and inputs $I D_{i}$ and $P W_{i}$.

2) The smart card computes $\mathrm{A}_{\mathrm{i}}=\mathrm{h}\left(\mathrm{b} \oplus \mathrm{PW}_{\mathrm{i}}\right), \mathrm{C}_{\mathrm{i}}^{*}=\mathrm{h}\left(\mathrm{ID}_{\mathrm{i}}\|\mathrm{h}(\mathrm{y})\| \mathrm{A}_{\mathrm{i}}\right)$, and checks whether the computed $\mathrm{C}_{\mathrm{i}}^{*}$ is equal to $C_{i}$. If they are not equal, the smart card rejects the password change request. Otherwise, the user $U_{i}$ inputs a new password $\mathrm{PW}_{\text {new }}$ and a new random number $\mathrm{b}_{\text {new }}$.

3) The smart card computes $A_{\text {inew }}=h\left(b_{\text {new }} \oplus P W_{\text {new }}\right)$ and $C_{\text {inew }}=h\left(I D_{i}\|h(y)\| A_{\text {inew }}\right)$.

4) Finally, the smart card replaces $C_{i}$ with $C_{\text {inew }}$ to finish the password change phase.

\section{Cryptanalysis of Li et al.'s Protocol}

In this section, we demonstrate that Li et al.'s protocol is vulnerable to replay attack, denial of service attack, smart card lost attack, eavesdropping attack and server spoofing attacks.

\subsection{Vulnerable to Replay Attack}

Assume that a malicious attacker can eavesdrop the communication channel and intercepts the message $\left\{\mathrm{P}_{\mathrm{ij}}\right.$, $\mathrm{CID}_{\mathrm{i}}, \mathrm{M}_{1}, \mathrm{M}_{2}$ \}. Now if he resends this message, server $\mathrm{S}$ does not verify the freshness of nonce, $\mathrm{N}_{\mathrm{i}}$ and computes $\mathrm{N}_{\mathrm{i}}=\mathrm{h}\left(\mathrm{SID}_{\mathrm{j}} \| \mathrm{h}(\mathrm{y})\right) \oplus \mathrm{M}_{2}, \mathrm{E}_{\mathrm{i}}=\mathrm{P}_{\mathrm{ij}} \oplus \mathrm{h}\left(\mathrm{h}\left(\mathrm{SID}_{\mathrm{j}}\|\mathrm{h}(\mathrm{y})\| \mathrm{N}_{\mathrm{i}}\right), \mathrm{B}_{\mathrm{i}}=\mathrm{E}_{\mathrm{i}} \oplus \mathrm{h}(\mathrm{x} \| \mathrm{y}), \mathrm{D}_{\mathrm{i}}=\mathrm{h}\left(\mathrm{B}_{\mathrm{i}} \| \mathrm{h}(\mathrm{x} \| \mathrm{y})\right)\right.$ and $\mathrm{h}\left(\mathrm{P}_{\mathrm{ij}}\left\|\mathrm{CID}_{\mathrm{i}}\right\| \mathrm{D}_{\mathrm{i}} \| \mathrm{N}_{\mathrm{i}}\right)$ and compares with $\mathrm{M}_{1}$. The condition satisfies and $\mathrm{S}$ accepts the login request.

Now, $S$ computes $M_{3}=h\left(D_{i}|| A_{i} \| N_{j}|| S I D_{j}\right), M_{4}=A_{i} \oplus N_{i} \oplus N_{j}$ and sends $\left\{M_{3}, M_{4}\right\}$ to $U_{i}$. Here the attacker cannot find $\mathrm{N}_{\mathrm{j}}$ but he is successful in wasting server's valuable computing resources. A large number of replay attacks launched at the same time can also form denial-of-service attack.

\subsection{Vulnerable to Denial-of-Service Attack}

$\mathrm{n}$ active attacker who is also a valid user knowing $\mathrm{h}(\mathrm{y})$ can fabricate the message $\mathrm{M}_{2}$ using different nonce, say $\mathrm{N}_{\mathrm{A}}$ and sends the fabricated message $\left\{\mathrm{P}_{\mathrm{ij}}, \mathrm{CID}_{\mathrm{i}}, \mathrm{M}_{1}, \mathrm{M}_{2}\right\}$ to server, $\mathrm{S}_{\mathrm{j}}$ where $\mathrm{M}_{2}=\mathrm{h}\left(\mathrm{SID}_{\mathrm{j}} \| \mathrm{h}(\mathrm{y})\right) \oplus \mathrm{N}_{\mathrm{A}}$. After 
performing the steps mentioned in 2.3.1, server $S_{j}$ rejects the login request of $U_{i}$, who is a legitimate user, as $\mathrm{h}\left(\mathrm{P}_{\mathrm{ij}}|| C \mathrm{CID}_{\mathrm{i}}|| \mathrm{D}_{\mathrm{i}}|| \mathrm{N}_{\mathrm{i}}\right)$ does not equal to the received $\mathrm{M}_{1}$. Hence, denial-of-service attack is possible.

\subsection{Vulnerable to Smart Card Lost Attack and Password Guessing Attack}

Assume that the user's smart card has been lost or stolen. The attacker can extract the information $\mathrm{C}_{\mathrm{i}, \mathrm{D}} \mathrm{D}_{\mathrm{i}} \mathrm{E}_{\mathrm{i}}, \mathrm{h}($.), $h(y)$, b from the smart card $[20,21]$. By previously intercepted message, attacker can find $N_{i}, E_{i}$ using the following calculations.

$\mathrm{N}_{\mathrm{i}}=\mathrm{M}_{2} \oplus \mathrm{h}\left(\mathrm{SID}_{\mathrm{j}} \| \mathrm{h}(\mathrm{y})\right)$,

$\mathrm{E}_{\mathrm{i}}=\mathrm{P}_{\mathrm{ij}} \oplus \mathrm{h}\left(\mathrm{h}\left(\mathrm{SID}_{\mathrm{j}} \| \mathrm{h}(\mathrm{y})\right) \| \mathrm{N}_{\mathrm{i}}\right)$

Now, $A_{i}=C_{i} \oplus h\left(D_{i}\left\|S_{i} D_{j}\right\| N_{i}\right)$.

Using offline dictionary attack, attacker can find the ID, password PW of $\mathrm{U}_{\mathrm{i}}$ by performing following operations:

1) Compare $C_{i}$ with $h\left(I D_{\text {guess }}\|h(y)\| A_{i}\right)$. Whenever it equals, $I D_{\text {guess }}$ is $I_{\mathrm{i}}$ of the user $U_{i}$.

2) Compare $A_{i}$ with $\mathrm{h}\left(\mathrm{b} \oplus \mathrm{PW}_{\text {guess }}\right)$.Whenever it equals, $P \mathrm{PW}_{\text {guess }}$ is the original $\mathrm{PW}$ of $\mathrm{U}_{\mathrm{i}}$.

As the ID and Password are known, attacker can use the smart card impersonating the original user.

\subsection{Vulnerable to Eavesdropping Attack}

Assume that attacker found the smart card details. He can intercept the message $\left\{\mathrm{P}_{\mathrm{ij}}, \mathrm{CID}_{\mathrm{i}}, \mathrm{M}_{1}, \mathrm{M}_{2}\right\}$. He can find $\mathrm{N}_{\mathrm{i}}=\mathrm{h}\left(\operatorname{SID}_{\mathrm{j}} \| \mathrm{h}(\mathrm{y})\right) \oplus \mathrm{M}_{2}$ and $\mathrm{A}_{\mathrm{i}}=\mathrm{ID}_{\mathrm{i}} \oplus \mathrm{h}\left(\mathrm{D}_{\mathrm{i}}\left\|\mathrm{SID}_{\mathrm{j}}\right\| \mathrm{N}_{\mathrm{i}}\right)$, intercepts the message $\left\{\mathrm{M}_{3}, \mathrm{M}_{4}\right\}$ from server and computes $N_{j}=A_{i} \oplus N_{i} \oplus M_{4}$. Now he acquires the session key, $S K=h\left(D_{i} \| A_{i}|| N_{i}|| N_{j}|| S I D_{j}\right)$. Hence, the entire communication is compromised using this passive attack as the attacker has known the session key.

\subsection{Vulnerable to Server Spoofing Attack}

If we assume the attacker, $A$ broke into a server or acquired a malicious server, then attacker have $\mathrm{h}(\mathrm{x} \| \mathrm{y})$ and $\mathrm{h}\left(\mathrm{SID}_{\mathrm{j}} \| \mathrm{h}(\mathrm{y})\right)$. Attacker, A can masquerade as server, $\mathrm{S}_{\mathrm{j}}$ to spoof user, $\mathrm{U}_{\mathrm{i}}$.

After intercepting the login request message $\left\{\mathrm{P}_{\mathrm{ij}}, \mathrm{CID}_{\mathrm{i}}, \mathrm{M}_{1}, \mathrm{M}_{2}\right\}$, A can compute $\mathrm{N}_{\mathrm{i}}=\mathrm{h}\left(\operatorname{SID}_{\mathrm{j}} \| \mathrm{h}(\mathrm{y}) \oplus \mathrm{M}_{2}, \mathrm{E}_{\mathrm{i}}\right.$ $=\mathrm{P}_{\mathrm{ij}} \oplus \mathrm{h}\left(\mathrm{h}\left(\operatorname{SID}_{\mathrm{j}} \| \mathrm{h}(\mathrm{y})\right) \| \mathrm{N}_{\mathrm{i}}\right), \mathrm{B}_{\mathrm{i}}=\mathrm{E}_{\mathrm{i}} \oplus \mathrm{h}(\mathrm{x} \| \mathrm{y}), \mathrm{D}_{\mathrm{i}}=\mathrm{h}\left(\mathrm{B}_{\mathrm{i}} \| \mathrm{h}(\mathrm{x} \| \mathrm{y})\right), \mathrm{A}_{\mathrm{i}}=\mathrm{CID}_{\mathrm{i}} \oplus \mathrm{h}\left(\mathrm{D}_{\mathrm{i}}\left\|\operatorname{SID}_{\mathrm{j}}\right\| \mathrm{N}_{\mathrm{i}}\right)$. A can choose a nonce, $N_{A}$ and compute $M_{3}=h\left(D_{i}\left\|A_{i}\right\| N_{A} \| S_{D}\right), M_{4}=A_{i} \oplus N_{i} \oplus N_{A}$. A then sends the message $\left\{M_{3}, M_{4}\right\}$ to user $U_{i}$ masquerading as server $S_{j}$. $U_{i}$ computes $N_{A}=A_{i} \oplus N_{i} \oplus M_{4}$, and compares $M_{3}$ with $h\left(D_{i}\left\|A_{i}\right\| N_{A} \| S D_{j}\right)$. Then $\mathrm{U}_{\mathrm{i}}$ computes mutual authentication message $\mathrm{M}_{5}=\mathrm{h}\left(\mathrm{D}_{\mathrm{i}}\left\|\mathrm{A}_{\mathrm{i}}\right\| \mathrm{N}_{\mathrm{i}}|| \mathrm{SID}_{\mathrm{j}}\right)$ and sends to attacker, $A$ who is masquerading as $S_{j}$. Then A verifies $M_{5}$ and mutual authentication is done. Finally attacker, $A$ and User, $U_{i}$ computes the session key, $\mathrm{SK}=\mathrm{h}\left(\mathrm{D}_{\mathrm{i}}\left\|\mathrm{A}_{\mathrm{i}}|| \mathrm{N}_{\mathrm{i}}|| \mathrm{N}_{\mathrm{A}}\right\| \mathrm{SID}_{\mathrm{j}}\right)$.

\section{Conclusion}

In this paper, we have shown that Li et al.'s dynamic ID based authentication protocol cannot resist many attacks and is vulnerable to replay attack, denial of service attack, smart card lost attack, eavesdropping attack and server spoofing attacks. We strongly feel that a remote user authentication protocol should provide security against the above mentioned attacks so that it can be used in the real world applications.

\section{References}

[1] Hsiang, H. and Shih, W. (2009) Weaknesses and IMPROVEMENTs of the Yoon-Ryu-Yoo Remote User Authentication Scheme Using Smart Cards. Computer Communications, 32, 649-652. http://dx.doi.org/10.1016/j.comcom.2008.11.019

[2] Yoon, E.J., Ryu, E.K. and Yoo, K.Y. (2004) Further Improvement of An Efficient Password Based Remote User Authentication Scheme Using Smart Cards. IEEE Transactions on Consumer Electronics, 50, 612-614. http://dx.doi.org/10.1109/TCE.2004.1309437

[3] Wang, X., Zhang, W., Zhang, J. and Khan, M.K. (2007) Cryptanalysis and Improvement on Two Efficient Remote User Authentication Scheme Using Smart Cards. Computer Standards and Interfaces, 29, 507-512. http://dx.doi.org/10.1016/j.csi.2006.11.005

[4] Lee, C.C., Lai, Y.M. and Li, C.T. (2012) An Improved Secure Dynamic ID Based Remote User Authentication 
Scheme for Multi-Server Environment. International Journal of Security and Its Applications, 6, 203-209.

[5] Sood, S.K., Sarje, A.K. and Singh, K. (2011) A Secure Dynamic Identity Based Authentication Protocol for MultiServer Architecture. Journal of Network and Computer Applications, 34, 609-618. http://dx.doi.org/10.1016/j.jnca.2010.11.011

[6] Guo, D.L. and Wen, F.T. (2013) A More Secure Dynamic ID Based Remote User Authentication Scheme for MultiServer Environment. Journal of Computational Information Systems, 9, 407-414.

[7] Madhusudhan, R. and Mittal, R.C. (2012) Dynamic ID-Based Remote User Password Authentication Schemes Using Smart Cards: A Review. Journal of Network and Computer Applications, 35, 1235-1248. http://dx.doi.org/10.1016/j.jnca.2012.01.007

[8] Chena, T.-H., Hsiang, H.-C. and Shih, W.-K. (2011) Security Enhancement on an Improvement on Two Remote User Authentication Schemes Using Smart Cards. Future Generation Computer Systems, 27, 377-380. http://dx.doi.org/10.1016/j.future.2010.08.007

[9] Fan, C.I., Chan, Y.C. and Zhang, Z.K. (2005) Robust Remote Authentication Scheme with Smart Cards. Computers \& Security, 24, 619-628. http://dx.doi.org/10.1016/j.cose.2005.03.006

[10] Lin, I.C., Hwang, M.S. and Li, L.H. (2003) A New Remote User Authentication Scheme for Multi-Server Architecture. Future Generation Computer Systems, 19, 13-22. http://dx.doi.org/10.1016/S0167-739X(02)00093-6

[11] Liao, I.E., Lee, C.C. and Hwang, M.S. (2006) A Password Authentication Scheme over Insecure Networks. Journal of Computer and System Sciences, 72, 727-740. http://dx.doi.org/10.1016/j.jcss.2005.10.001

[12] Li, X., Xiong, Y.P., Ma, J. and Wang, W.D. (2012) An Efficient and Security Dynamic Identity Based Authentication Protocol for Multi-Server Architecture Using Smart Cards. Journal of Network and Computer Applications, 35, 763769. http://dx.doi.org/10.1016/j.jnca.2011.11.009

[13] Chang, C.C. and Lee, J.S. (2004) An Efficient and Secure Multi-Server Password Authentication Protocol Using Smart Cards. Proceedings of the Third International Conference on Cyberworlds, November, 417-422.

[14] Tsaur, W.J., Wu, C.C. and Lee, W.B. (2004) A Smart Card-Based Remote Scheme for Password Authentication in Multi-Server Internet Services. Computer Standards \& Interfaces, 27, 39-51. http://dx.doi.org/10.1016/j.csi.2004.03.004

[15] Tsai, J.L. (2008) Efficient Multi-Server Authentication Scheme Based on One-Way Hash Function Without Verification Table. Computers \& Security, 27, 115-121. http://dx.doi.org/10.1016/j.cose.2008.04.001

[16] Liao, Y.P. and Wang, S.S. (2009) A Secure Dynamic ID Based Remote User Authentication Scheme for Multi-Server Environment. Computer Standards \& Interfaces, 31, 24-29. http://dx.doi.org/10.1016/j.csi.2007.10.007

[17] Hsiang, H.C. and Shih, W.K. (2009) Improvement of the Secure Dynamic ID Based Remote User Authentication Scheme for Multi-Server Environment. Computer Standards \& Interfaces, 31, 1118-1123. http://dx.doi.org/10.1016/j.csi.2008.11.002

[18] Lee, C.C., Lin, T.H. and Chang, R.X. (2011) A Secure Dynamic ID Based Remote User Authentication Scheme for Multi-Server Environment Using Smart Cards. Expert Systems with Applications, 38, 13863-13870.

[19] Li, X., Ma, J., Wang, W.D., Xiong, Y.P. and Zhang, J.S. (2013) A Novel Smart Card and Dynamic ID Based Remote User Authentication Scheme for Multi-Server Environments. Mathematical and Computer Modelling, 58, 85-95. http://dx.doi.org/10.1016/j.mcm.2012.06.033

[20] Kocher, P., Jaffe, J. and Jun, B. (1666) Differential Power Analysis, Advances in Cryptology. Proceedings of CRYPTO’99, LNCS, 1999, 388-397

[21] Messaerges, T.S., Dabbish, E.A. and Sloan, R.H. (2002) Examining Smart Card Security under the Threat of Power Analysis Attacks. IEEE Transactions on Computers, 51, 541-552. http://dx.doi.org/10.1109/TC.2002.1004593 